\title{
Methylprednisolone was associated with an increase in death after head injury
}

CRASH Trial Collaborators. Effect of intravenous corticosteroids on death within 14 days in 10008 adults with clinically significant head injury (MRC CRASH trial): randomised placebo-controlled trial. Lancet 2004;364:1321-8.

In patients with head injury, is early administration of methylprednisolone better than placebo for reducing death?

METHODS

Design: randomised placebo controlled trial (Corticosteroid
Randomisation After Significant Head injury [CRASH]).

\section{MAIN RESULTS}

The first patient was enrolled in April 1999. Recruitment was stopped in May 2004. At 2 weeks, all cause mortality was greater in patients who received methylprednisolone than in those who received placebo (table). Preplanned subgroup analysis based on time from injury to

For correspondence: CRASH Trials Coordinating Centre, London School of Hygiene and Tropical Medicine, London, UK. crash@|shtm.ac.uk

Sources of funding: UK Medical Research Council, Pharmacia and Upjohn.

Methylprednisolone (MP) $v$ placebo for head injury at 2 weeks*

\begin{tabular}{lllll}
\hline Outcome & MP & Placebo & RRI $(95 \%$ CI) & NNH (CI) \\
\hline $\begin{array}{l}\text { All cause } \\
\text { mortality }\end{array}$ & $21 \%$ & $18 \%$ & $18 \%(9$ to 27$)$ & 32 (22 to 63) \\
\hline
\end{tabular}

*Abbreviations defined in glossary; $\mathrm{RRI}, \mathrm{NNH}$, and $\mathrm{Cl}$ calculated from data in article. randomisation ( $\leqslant 1 \mathrm{~h},>1$ to $\leqslant 3 \mathrm{~h}$, or $>3$ to $\leqslant 8 \mathrm{~h}$ ) and severity of injury (GCS mild [ 13 to 14 ], moderate [9 to 12 ], or severe [3 to 8 ]) showed that groups did not differ for mortality according to time since injury $(\mathrm{p}=0.05)$ or injury severity $(\mathrm{p}=0.22)$.

\section{CONCLUSION}

In patients with head injury, administration of 48 hours of methylprednisolone was associated with an increase in all cause mortality at 2 weeks.

A modified version of this abstract appears in ACP Journal Club.

\section{Commentary}

purred by the 1997 Cochrane review, ${ }^{1}$ weak supporting evidence, and continued clinical use, the CRASH trial evaluated the effects of (TBI) high dose corticosteroids on mortality after traumatic brain injury

Strengths of the study include enrolment of $>10000$ patients from 49 countries, concealed allocation, and intention to treat analysis. A minor weakness of the study is that cause of death was not adjudicated. In individual cases, death could be due to causes not attributable to corticosteroid use. However, randomisation in a trial of this size would account for any random variation between groups, and thus the increase in mortality is attributable to the only systematic difference between the 2 groups - the use of corticosteroids. Inclusion of additional baseline information, such as blood alcohol concentration or glycaemic control, would have been useful to further demonstrate balance between groups, but systematic variation influencing the outcome is unlikely.

One of the reasons cited for the failure of most clinical trials in TBI from the bench to the bedside is that they are not structured for level of injury. ${ }^{2}$ In a trial of this size, inclusion of the spectrum of TBI patients could be criticised, but the distribution of severity was balanced, and increased mortality was not associated with the range of severity, especially in moderate and severe TBI. Patients receiving corticosteroids were also more likely to die across the range of clinical signs seen in TBI.

The surprising result of the CRASH trial shows yet again that strong evidence is a categorical imperative in health care and should reinforce that "flying blind" when caring for patients is quite unsafe. The lack of a plausible biological explanation for the difference in mortality rates should not be thought to refute the evidence. Early administration of high dose corticosteroids increases the risk of death when used to treat TBI, and routine use should be discontinued.

Hilaire J Thompson, RN, CNRN, PhD School of Nursing, University of Washington Seattle, Washington, USA Asha Bakshi, MD

Department of Neurosurgery, University of Pennsylvania Philadelphia, Pennsylvania, USA

1 Alderson P, Roberts I. Corticosteroids in acute traumatic brain injury: systematic review of randomised controlled trials. BMJ 1997;314:1855-9.

2 Bullock MR, Lyeth BG, Muizelaar JP. Current status of neuroprotection trials for traumatic brain injury: lessons from animal models and clinical studies. Neurosurgery 1999;45:207-17. 